\title{
ANALYSIS OF FREQUENCY ON A LARGE INTERCONNECTED SYSTEM
}

\author{
par CARROLL F. MERRIAM
}

Ingénieur à la Pensylvania WATER-POWER Co

In a recent conference concerning the possibilities of using the rate of change of frequency in contradistinction to frequency alone as an element in governing, it became apparent that those present had little idea as to the actuai magnitudes of frequency variations or rates of change that would be encountered on a large interconnected system.

Consequently, M. JE ALLEN, Chief of Tests for the Pennsylvania Water $\&$ Power Company had $\mathrm{Mr}$. Wm. B. HESS take some snap samples of the frequency at Safe Harbor using the most expanded scale possible with the present recorder. These samples consisted of a number of two minute runs during some of which the tie to the New-York system was closed while in others it was open. The results are shown in the heavy solid curve in the accompanying diagrams. These have been traced from the original records on which the instrument was given its maximum speed of paper travel, 12 inches per minute. The vertical scale is such that 1.6 inch represents a tenth of a cycle in 60 . Some idea of the magnification of this scale may be had by realizing that the zero would be approximately 80 feet of the paper. The 60 cycle line is not shown since the absolute value of frequency was not indicated, but rather the relative changes.

The curve as drawn by the recorder has been differentiated by M. Paul BOCK ans Miss Edith NAUKIWELL using a mirror, to determine the normal to the curve at frequent intervals. The normal was then converted graphically to slope, or rate of change, and plotted as the curve marked "Change in Frequency in Cycles per Second per Second $》$.

It is also interesting to see to what extent the integrated frequency would wander from the average frequency during each two minute run. The result of this integration can be expressed as phase shift in electrical degrees, as shown by the curve marked «Accumulation of Frequency in Cycles».

This test undoubtedly is a fair sample of the conditions to be met in normal operation on a very large interconnected system. The runs were made on a weekday afternoon to avoid the extreme conditions when an unusually large amount of generatior. capacity would be on the line over the peak hour, or when the system would be lightly loaded during the night valley. The total in interconnected capacity at the time these tests were made is estimated to be approximately 5 millions $\mathrm{kw}$. without the New-York tie closed, and 11 millions $\mathrm{kw}$. with the interconnection to New-York and New-England

Theoretically the frequency curve on any system is the résultant of subtraction from and additions to the kinetic energy contained in the sum total of all rotating equipment, both motors and generators, connected to the system. The subtractions are caused by increases in load taken by the consumers, or by reduction in the generation when the governors of the prime movers respond to high frequency. In like manner the additions are caused by load rejected by the consumers, or to picking up of load by the generating units when the frequency is low. The effect of load changes on the system may be assumed to be very nearly purely statistical over short periods of time, while the effect or response of the prime mover governors should be a function of the frequency. Considering the erratic behaviour that has been observed on many governors there is question as to whether even this element may not be to a large extent accidental

The theoretical effect of sudden applications and rejections of load in a purely accidental manner would be a jagged market prices. Here a myriad of complex reactions combine to throw prices up or down apparently without advance warning. 
One of the most significant facts brought out by these tests is that in spite of the fact that the instrument was perfectly capable of recording rates on change in frequency up to 1/3 of a cycle per second per second, or about five times the maximum rate of change observed, nevertheless all of the fluctuations have definitely a sinusoida form. When a wave of this kind is differentiated the result is another curve also sinusoidal in form, but advanced in phase by 90 degrees.

The absence of the jagged wave form is an evidence of the elastic nature of electrical transmission systems. The sudden application of a load at one point, which has been shown by M. ALLEN to produce a sudden and sharp jog in the frequency at that particular location, quickly becomes dulled as the frequency disturbance radiates outward into the system as a whole. Consequently, prime movers, particularly those at a distance from the load change, do not feel the sudden application that is assumed in the usual mathematical analyses of governor problems. Instead they feel the same effect as though the power were transmitted to the point of load application through a long flexible shaft.

This has an important bearing upon the practicability of applying « inertia» or its equivalent in the operation of prime mover governors. According to the classic mathematical analysis referred to above, the acceleration at moment of load application may appear to be so drastic that it would be likely to lead to jumpy action of the governor. Furthermore, since the departure from zero acceleration starts at the same instant that the frequency itself departs from normal, the gain in time resulting from the fact that acceleration leads the frequency is not large. Consequently, under such theoretical conditions, the value of inertia governing would seem to be questionable On the other hand, if we consider the true nature of what is happening in normal operation, we see that the use of acceleration as a means of causing the governor to anticipate the impeding trend in frequency presents a definite prospect of stabilising the governor action.

The purpose of taking these records is to give those who may be interested in applying this principle an opportunity to gauge the magnitude of the forces that might be senerated by whatever device might be used to feed the correcting impulse to the governor. This analysis may be of value to those considering electronic devices rather than mechanical for accomplishing the same result.

In evaluating the benefits to be derived from the use of such developments, it should be remembered that the objective is not to approach perfection in the appearance of the frequency record, or to establish in our power systems a time standard of astronomical quality. Pratical perfection has already been attained in this respect merely by the largeness of interconnected systems, and so is no longer of vital concern to the power companies.

The real value lies in the fact that the the acceleration wave travels outward from the point of load change and, therefore, will be felt first by the prime movers which are nearer at hand. If the governors of these units can be made to feel and act upon the reception of this wave, its outward progress will be retarded. The result will be that inter-regional transmission lines will then be less subject to load fluctuations, because the prime movers in each load center will be more alert to respond to the load changes originating within their own particular spheres of influence. 


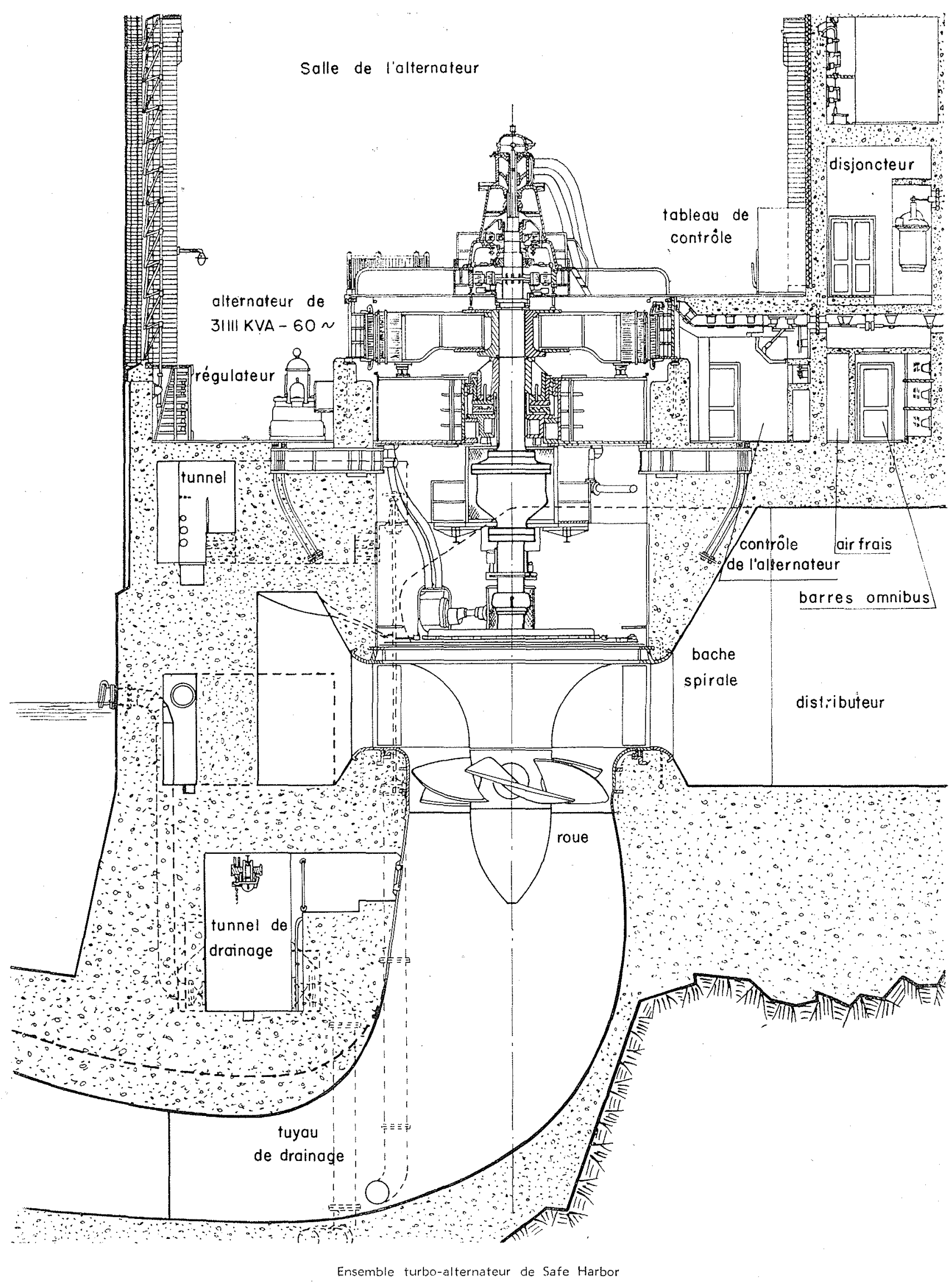




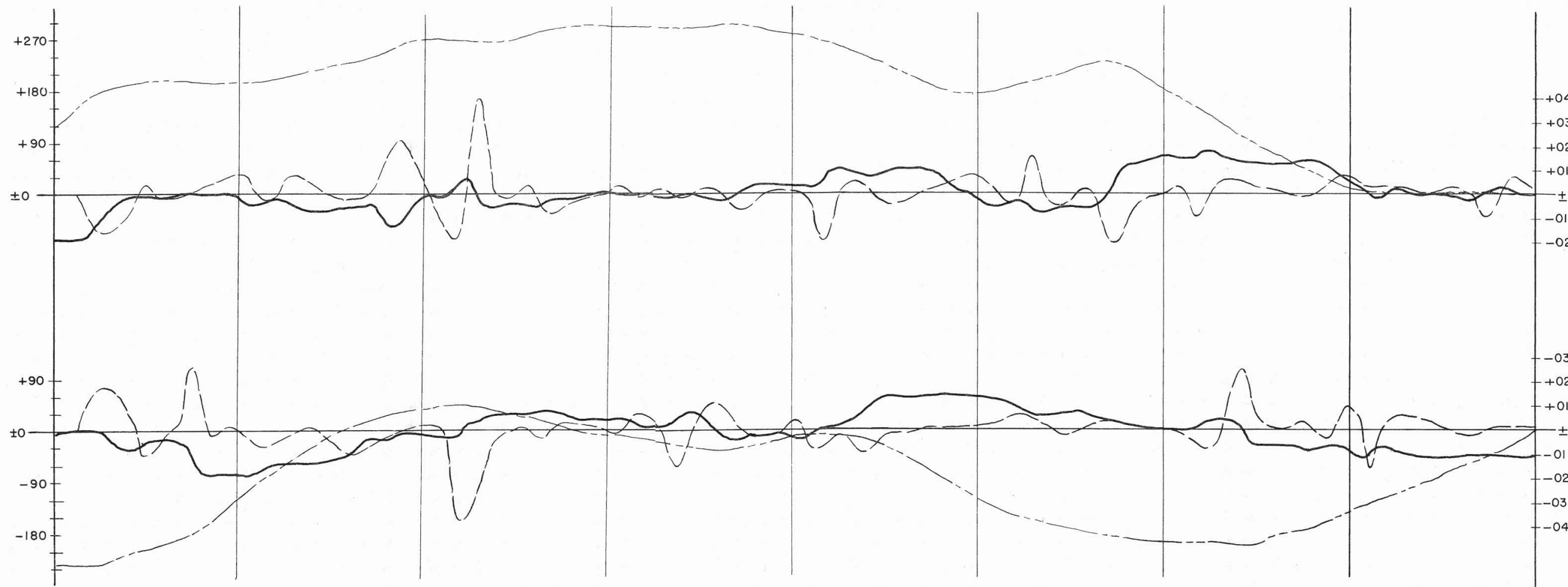




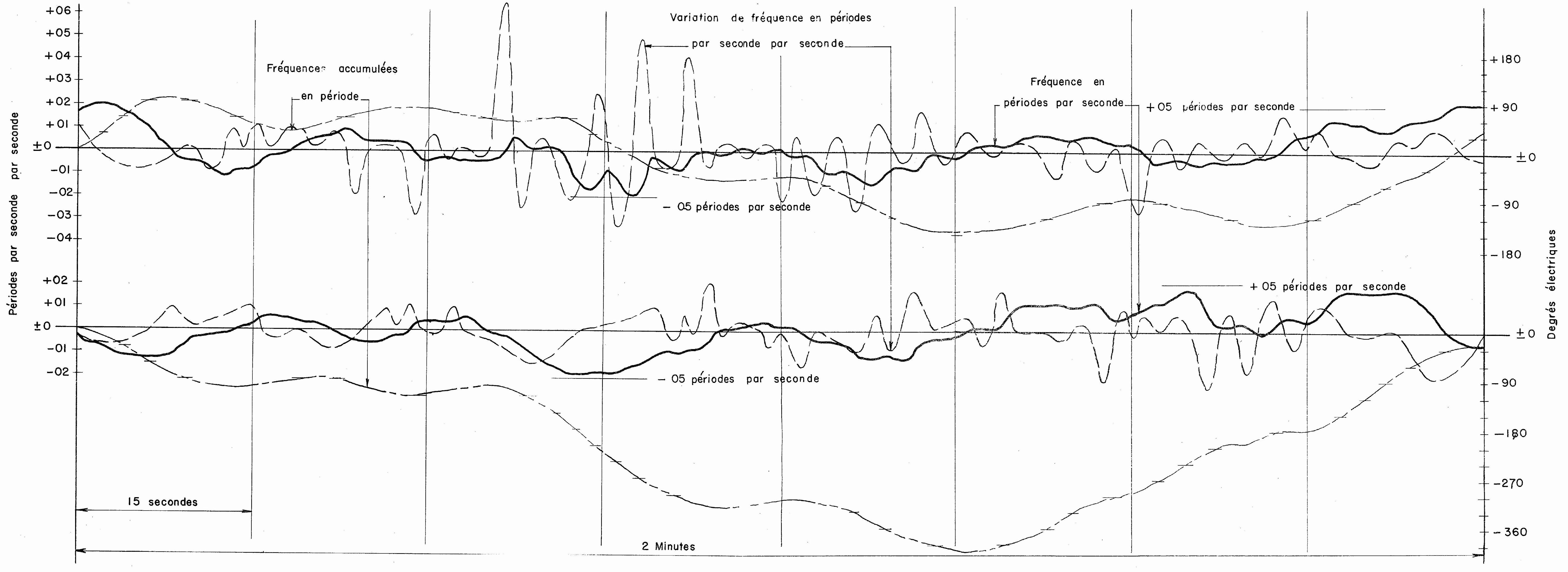


